\title{
Political Uncertainty and Crime in Transition Economies*
}

\author{
Barbara G. Katz \\ Stern School of Business, New York University \\ 44 W. 4th St., New York, NY 10012 \\ bkatz@stern.nyu.edu; tel: 212998 0865; fax: 2120054218 \\ corresponding author \\ Joel Owen \\ Stern School of Business, New York University \\ 44 W. 4th St., New York, NY 10012 \\ jowen@stern.nyu.edu; tel: 212998 0446; fax: 2129954003 \\ January 21, 2007 \\ Preliminary and incomplete: Please do not quote
}




\section{Abstract \\ Political Uncertainty and Crime in Transition Economies}

Two stylized facts are often used to characterize the economies in transition: an increase in the crime level and frequent government changes, where the party in power is replaced by another party with a different, and often opposite, ideological orientation. We investigate the impact on agents' honesty when agents perceive the future form of government as uncertain, and also know that their own collective decisions will effect the government's choice of type. Furthermore, we assume that the form that the government will take depends, in part, on the collective behavior of the agents. By endogenizing the joint decisions made by the agents, as well as the government, we derive the social consequences of these choices, the induced level of crime. Using the level of crime permits us to investigate comparative statics for possible policy implications. We show that the complex interactions between the government and the agents leads to some non-intuitive results. 


\section{Introduction}

In the almost two decades since the fall of communism, governments in the economies in transition have changed frequently, becoming more or less democratic and more or less authoritarian. ${ }^{1}$ These changes in government were influenced, in part, by the reactions of agents within the society to government policies, as well as by the actions taken by the participants in the government itself. Agents were forced to make decision whose outcomes depended on the ensuing, but not completely known, form of government, whereas the ensuing form of government was influenced by the decisions of the agents. In this paper we investigate the impact on agents' honesty when agents perceive the future form of government as uncertain. Specifically, we study the impact of this uncertainty, which we call political uncertainty, on the social fabric of society as measured by its crime level.

Besides undergoing frequent changes in governments, often associated with reversals in ideological orientations, economies in transition are also characterized as having experienced increases in crime. The issue of crime in transition economies is widely discussed and well documented. ${ }^{2}$ Most models investigating the decisions of agents in transition economies to steal start with a benchmark case in which the agents face a known type of government. The agent optimizes given the existing government, and then the problem is re-solved under the assumption of a different form of government. The agents' decisions in the two situations are then compared. Although agents make decisions in two different contexts, there is no self-awareness on the part of the agent that the government might be of different types. Examples of such studies include Polishchuk and Savvateev (2004), Sonin (2003), and Katz

\footnotetext{
${ }^{1}$ See Kornai (2006) for a listing of the changes in governments in various economies in transition. Reversals of ideological orientation characterize these changes.

${ }^{2}$ See, for example, Lotspeich (1995) which notes empirical evidence relating to the increase in crime rates across transition economies, and considers various explanations for the phenomenon.
} 
and Owen (2005). A particular type of government, known to the agents, is also assumed in Grossman (1995) and Alexeev, Janeba and Osborne (2004), which focus on "mafias" competing with the state for entrepreneurial rents. Dixit (2004), which suggests a principal-agent model to capture the intent of a government to induce efficiency in society, also assumes a given governmental form, known to the agents. Hoff and Stiglitz (2004) take a different tack by assuming agents face uncertainty regarding the form of government that will arise. They endogenize the probability of occurrence of these governments using a consistency requirement among the agents, and find that the uncertainty of governmental form leads to multiple solutions for the crime level. Katz and Owen (2006) also consider a model with two governments. They endogenize the probability of occurrence based on the implied revenues each government might acquire and find a unique crime level. Neither Hoff and Stiglitz nor Katz and Owen allow the government to be an active participant in the choice of its form.

We contribute to the literature on the rule of law in transition by allowing both agents and the government to be active participants in the formation of the type of government that evolves, and show some of the social consequences of such a joint interaction. First, we permit the agents to make their decisions knowing not only that the future form of government is uncertain, but also knowing that their own collective decisions will effect the government's choice of type. Second, we assume that the form that the government will take depends, in part, on the collective behavior of the agents. By endogenizing the joint decisions made by the agents, as well as the government, we derive the social consequences of these choices, the level of crime induced. Using the level of crime permits us to investigate comparative statics for possible policy implications. We show that the complex interactions between the government and the agents leads to some non-intuitive results. We also contribute to the literature on the role of institutions in transition (for example, Djankov and 
Murrell (2002), McMillan (2002) and Bevan and Estrin (2004)), and to that stressing, more generally, that different economic outcomes are to be expected from different institutional arrangements (for example, Shleifer and Vishny (1998) and Acemoglu, Johnson and Robinson (2001, 2002, 2003)). We extend this literature by allowing agents to be aware that they face different institutional arrangements, and investigate the implications of this knowledge.

\section{Model}

To model the impact of changing governments on decisions made by agents, we assume that at the moment the agents' decisions are made, all agents believe that the present government can evolve into one of two possible forms. At some point in the future, the specific forms of government will be determined. The consequences of agents' decisions depend on which form the government takes, and are thus influenced by the uncertainty of this form, or what we call political uncertainty. On the other hand, the ultimate choice of governmental form depends on the collective decisions of the agents.

We designate the two possible forms of government as G1 and G2. G1 is a government which is concerned with maximizing welfare and reducing crime in society, albeit constrained by its tax revenues. G2 is a government which is interested in maximizing tax revenue. The restriction of the model to two future forms of government is an assumption of convenience. The two we have chosen have features that are meant to represent a democratic government, G1, and a more self-serving government, G2.

There is a continuum of agents, each of whom owns one firm. Since operating a firm honestly might turn out to be unrewarding if G2 were to come into existence, it could be beneficial for the agent to divert to himself some of the firm's funds to insure against this possibility. To that end, we assume that the agent must decide whether 
or not to steal assets from the firm, and if he chooses to steal, how much. Stealing, of course, carries the risk of being caught and being punished, with the punishment depending on the subsequent form of government.

We assume that at the outset, the value of each agent's firm is normalized to 1 . Agent $a$ must decide on the proportion $\tau_{a}, \tau_{a} \in[0,1]$, to steal from the firm. There is a known probability $\lambda, \lambda \in[0,1]$, that an agent who steals $\tau_{a}$ will be caught, and this probability is assumed common to both potential government types. We consider the outcomes for the agent under G1 first. If the agents is caught, then under G1, the agent must return the stolen funds to the government, plus pay an additional penalty. The part of the firm not stolen, which we assume to have been run honestly, will be taxed at the punitive rate $t+\delta, t+\delta \leq 1$, where $t, t \in[0,1]$, is the normal tax rate, and $\delta, \delta \in[0,1]$, is the addition to the normal tax rate as a consequence of the agent's having been caught stealing. Thus, agent $a$ retains $(1-t-\delta)\left(1-\tau_{a}\right)(1+r)$ of the firm at the end of the period, where $r \in \mathbb{R}$ represents the impact of economic growth generated by G1. If agent $a$ is not caught, then under G1 he keeps the stolen funds and the honestly run part of the firm is worth $(1-t)\left(1-\tau_{a}\right)(1+r)$. Turning to G2, if agent $a$ is caught stealing, then he is punished by having to pay the government the proportion $b, b \in[0,1]$, of the entire firm. If agent $a$ is not caught, under G2 he pockets $\tau_{a}$ and the remainder of the firm is honestly run and worth $(1-t)\left(1-\tau_{a}\right)$ at the end of the period. Notice that it is assumed that G2 does not create a climate which allows the value of the firm to change. We assume that the level of law enforcement is represented by $\lambda$, and that the agents understand that $r$ will be chosen optimally by G1, should it come into existence, and that $b$ will be chosen optimally by G2, should it come into existence. We further note that, since G1 is constrained by its tax revenue, and since tax revenue is determined by the collective decisions of the agents, it is possible that optimization by G1 might lead to a negative value for $r$.

The agents still must ascribe a probability to the coming into existence of G1 or 
G2. We let $\pi, \pi \in[0,1]$, represent this probability, and assume there is a linkage between $\pi$ and the level of honesty within a society. The collective decisions of all the agents determine the proportion of these agents who steal more than some amount, say $\gamma$, and we denote this proportion as $K(\gamma)$. This proportion, in turn depends on the agents' knowing $\pi$ and thus we can write $K(\gamma)$ as $K(\pi)$. We assume that $\pi$ will be determined in a way to satisfy $\pi=1-K(\pi)$.

We assume that each agent $a$ incurs a cost (real or psychic) of stealing $\tau_{a}$ proportion of the firm, and that this cost is of the form $a \tau_{a}^{2} / 2$. Thus, we are assuming that some agents find it easier to steal than others. Furthermore, we assume that the $a$ values which index the agents are distributed as $H(a), a \in[0,1]$, and that $H(a)$ is continuous.

The decision problem agent $a$ faces is illustrated in the following diagram, where $O_{1}=0+(1-t-\delta)\left(1-\tau_{a}\right)(1+r)-a \tau_{a}^{2} / 2, O_{2}=\tau_{a}+(1-t)\left(1-\tau_{a}\right)(1+r)-a \tau_{a}^{2} / 2$, $O_{3}=(1-b)-a \tau_{a}^{2} / 2$, and $O_{4}=\tau_{a}+(1-t)\left(1-\tau_{a}\right)-a \tau_{a}^{2} / 2$.

[Insert Figure 1]

Assuming that all the parameters are known to the agents, the expected value to agent $a$ of stealing $\tau_{a}$ is equal to

$$
\text { constant }+\tau_{a}\{(1-\lambda) t-\pi[(1-t-\delta \lambda) r+(1-t-\delta) \lambda]\}-a \tau_{a}^{2} / 2
$$

where the constant does not depend on $\tau_{a}$. Differentiation of this expression yields the optimum value of $\tau_{a}$, denoted by $\tau_{a}^{*}$, and which is summarized in the following proposition.

Proposition 1. $\tau_{a}^{*}=\left\{\begin{array}{cc}1 & 0 \leq a \leq V \\ \frac{V}{a} & 0<V<a \\ 0 & V \leq 0\end{array}\right.$

where $V=(1-\lambda) t-\pi[(1-t-\delta \lambda) r+(1-t-\delta) \lambda]$. 
We define the crime rate as the proportion of $a$ values such that $\tau_{a}^{*}>\gamma$. We denote this crime rate as $K(V \mid \gamma)$, and describe it in Proposition 2.

Proposition 2. $K(V \mid \gamma)=\left\{\begin{array}{cc}1 & 0 \leq \gamma \leq V \\ H\left[\frac{V}{\gamma}\right] & 0<V<\gamma \\ 0 & V \leq 0\end{array}\right.$

Proof. See Appendix.

It was assumed that $r$ and $b$ would be determined by G1 or G2, should they come into existence. We now address the evaluation of these variables. Assume the government does not know the individual choices of agents, but rather does know the decision of the representative (average) agent as well as a feature of the collective decisions of the agents, namely the crime level. In what follows, we denote by $\tau_{\bar{a}}^{*}$ the percentage of the firm stolen by the representative agent.

We begin with G1's problem. We assume that G1 chooses $r$ to maximize its benefits function $B_{1}(r)$, subject to a fiscal constraint. $B_{1}(r)$ has three components: agent $\bar{a}$ 's increased wealth, tax revenue and the level of honesty in society. Thus, we assume that $B_{1}(r)=$ [increased wealth $]+[$ tax revenue $]+[$ level of honesty $]$ subject to [tax revenue] $\geq[$ cost of government activities]. In particular, we let

$$
\begin{aligned}
& B_{1}(r)= {[(1-\lambda)] \tau_{\bar{a}}^{*}+(1-t)\left(1-\tau_{\bar{a}}^{*}\right)(1+r)-\lambda \delta\left(1-\tau_{\bar{a}}^{*}\right)(1+r) } \\
&\left.-\bar{a} \tau_{\bar{a}}^{*^{2}} / 2\right]+\left[\lambda \tau_{\bar{a}}^{*}+t\left(1-\tau_{\bar{a}}^{*}\right)+\lambda \delta\left(1-\tau_{\bar{a}}^{*}\right)\right](1+r) \\
&+k[1-K[V \quad \mid \quad \gamma]] \text { subject to }\left[\lambda \tau_{\bar{a}}^{*}+t\left(1-\tau_{\bar{a}}^{*}\right)+\lambda \delta\left(1-\tau_{\bar{a}}^{*}\right)\right](1+r) \geq c(r)
\end{aligned}
$$

where $k$ is the monetized marginal benefit the government gets from the level of honesty in society, and $c(r)$ is G1's cost of implementing policies that bring about a growth rate of $1+r$. The range of $r$ values over which $\mathrm{G} 1$ optimizes $B_{1}(r)$ is from the $r$ value that yields a totally crime-ridden society to the value that yields a totally law-abiding society. Specifically, let $r_{l}$ be the largest $r$ value satisfying $K(V \mid \gamma)=1$ 
and $r_{u}$ be the smallest $r$ value satisfying $K(V \mid \gamma)=0$. Thus, the problem facing G1 is to choose $r^{*}$ to satisfy

$$
r^{*}=\arg \max _{r_{l} \leq r \leq r_{u}} B_{1}(r) \text { such that }(\lambda-t-\lambda \delta) \tau_{\bar{a}}^{*}+t+\lambda \delta \geq c(r) /(1+r) .
$$

Should G2 come into existence, since it is only concerned with maximizing its tax revenue, it determines $b^{*}$ to satisfy

$$
\left.b^{*}=\arg \max _{0 \leq b \leq 1} B_{2}(b) \text { where } B_{2}(b)=\lambda b+(1-\lambda)\left(1-\tau_{\bar{a}}^{*}\right)() 1-t\right)
$$

Before we can solve the either G1 or G2's problem, we need to establish the level of crime induced by these policies. Thus, we begin with the agents' problems. For the remainder of the paper we assume that the distribution of agents, $H(a)$, is uniform. That is, we assume $H(a)=a$ for $a \in[0,1], H(a)=0$ for $a<0$ and $H(a)=1$ for $a>1$. Furthermore, we define $L=(1-\lambda) t$ and $M=(1-t-\delta \lambda) r+(1-t-\delta) \lambda$. In the next proposition we determine $\pi^{*}$.

Proposition 3. a. If $M>0$ and if $0<\gamma \leq L-M$, then $\pi^{*}=0$.

b. If $0<M<L<\gamma$, then $\pi^{*}=(\gamma-L) /(\gamma-M)$.

Proof. See Appendix.

We have excluded from consideration the case $V<0$, which would have led to $\pi^{*}=1$, implying certainty about the future form of government. We excluded this case because we are interested in the implications of political uncertainty, specifically, the uncertainty about the future form of government. For the remainder of the paper, we will exclude the case $\pi^{*}=0$, for the same reason.

Since the government uses the typical agent $\bar{a}$ to formulate its policy, we next establish how much the typical agent will steal from his firm.

Proposition 4. If $0<M<L<\gamma<\bar{a}$, then $\tau_{\bar{a}}^{*}=\frac{\gamma}{\bar{a}} \frac{L-M}{\gamma-M}$. 
Proof. See Appendix.

We now return to the government's problem. Recall that $\bar{a}$ is the value of $a$ attributed to the representative agent by the government in its calculations.

Earlier (Eq. 2), we established the problem that the government faced in determining the optimum value of $r, r^{*}$, if its form were G1. The next proposition provides the solution to this problem.

Proposition 5. a. Under the condition of P4, G1 chooses $r^{*}$ as the largest value of $r \in\left[r_{l}, r_{u}\right]$ such that $(\lambda-t-\lambda \delta) \tau_{\bar{a}}^{*}+t+\lambda \delta \geq c(r) /(1+r)$.

b. Let $\lambda>t+\lambda \delta$. If $c\left(r_{l}\right) /\left(1+r_{l}\right)<\lambda$ and $c\left(r_{u}\right) /\left(1+r_{u}\right) \geq t+\lambda \delta$, then $r^{*} \in\left(r_{l}, r_{u}\right)$.

c. Let $\lambda<t+\lambda \delta$. If $c\left(r_{l}\right) /\left(1+r_{l}\right)>\lambda$ and $c\left(r_{u}\right) /\left(1+r_{u}\right) \leq t+\lambda \delta$, then $r^{*} \in\left(r_{l}, r_{u}\right)$.

Proof. See Appendix.

P5 establishes that the optimal value $r^{*}$ is determined solely by the boundary (fiscal) constraint. Parts b and c provide conditions on the parameters that lead to an internal solution, i.e., a result in which neither everyone in society is honest nor everyone is dishonest. The conditions in parts b and c that lead to a non-extreme crime level can be interpreted as describing a tension between the probability of being caught and the tax plus penalty. For example, in part b, $\lambda$ is assumed to be large (relative to $c\left(r_{l}\right) /\left(1+r_{l}\right)$ ). A larger probability of being caught should induce an agent not to steal. But at the same time, the tax plus penalty is assumed to be small (relative to $c\left(r_{u}\right) /\left(1+r_{u}\right)$ ). A small penalty is an inducement to take a chance on being caught stealing. Part $\mathrm{c}$ has a similar interpretation. The two remaining cases, $\lambda$ large and tax plus penalty large and $\lambda$ small and tax plus penalty small, are not treated here since they lead to the extreme description of society as being composed of either all thieves or no thieves.

We also established in Eq. 4 the problem that the government faced in determining the optimal value of $b, b^{*}$, if the government's form were G2. The next proposition 
provides the solution to this problem.

Proposition 6. G2 chooses $b^{*}=1$.

Proof. See Appendix.

Having determined $r^{*}$ and $b^{*}$, we can evaluate the agents' decisions and determine the level of crime induced by the agents' uncertainty over which form of government will come into being. In particular, we can evaluate $V$ and $\pi$ at these optimal values, and when we do, we denote $V$ as $V^{*}$ and $\pi$ as $\pi^{*}$. We next examine the impact on $K\left(V^{*} \mid \gamma\right)$ of changes in the parameter values.

We focus our investigation of the comparative statics of our model on the conditions of parts b and c of P5. In so doing, we exclude the less interesting cases when the result of the choices made lead to a society in which either everyone steals or no one steals. The fact that $r^{*}>r_{l}$ implies that $V^{*}>0$, i.e., there will be some resulting crime. Furthermore, P5 parts b and c imply that, at optimality, the revenue constraint will be binding on G1, that is, $\tau_{\bar{a}}^{*}=\left[\frac{c\left(r^{*}\right)}{1+r^{*}}-t-\lambda \delta\right] /(\lambda-t-\lambda \delta)$. By the assumption of parts b and $\mathrm{c}$, the right-hand-side of this equation is a positive fraction so $\tau_{\bar{a}}^{*}=\frac{V}{\bar{a}}<1$. Finally, since $\bar{a}>\gamma$, it follows from P2 that $K\left(V^{*} \mid \gamma\right)=H\left(\frac{V^{*}}{\gamma}\right)$. Since $H\left(\frac{V^{*}}{\gamma}\right)$ is a monotonically increasing in $V^{*}$, directional changes in $K\left(V^{*} \mid \gamma\right)$ are the same as those for $V^{*}$. Let the right-hand-side of this revenue equation be denoted by $R\left(\theta, r^{*}\right)$, i.e., $R\left(\theta, r^{*}\right)=\left[\frac{c\left(r^{*}\right)}{1+r^{*}}-t-\lambda \delta\right] /(\lambda-t-\lambda \delta)$, where $\theta$ represents one of the parameters $\lambda, t$, or $\delta$. Note further that $r^{*}$ is also a function of $\theta$. In what follows, the partial derivatives are denoted by the appropriate subscript.

Proposition 7. Under the conditions of P5 parts $b$ and $c$

a. $\pi_{\theta}^{*}=\frac{1}{\gamma-M}\left[-L_{\theta}+M_{\theta} \pi^{*}\right]$.

b. $\tau_{\bar{a}_{\theta}}^{*}=\frac{\gamma}{\bar{a}}\left[\frac{1}{\gamma-M}\right]\left[L_{\theta}-M_{\theta} \pi^{*}\right]$.

c. $r_{\theta}^{*}=\frac{L_{\theta}-M_{\theta} \pi^{*}-\frac{\bar{a}}{\gamma}(\gamma-M) R_{\theta}}{M_{r^{*}} \pi^{*}+\frac{\bar{a}}{\gamma}(\gamma-M) R_{r^{*}}}$.

d. $V_{\theta}^{*}=\bar{a}\left[R_{\theta}+R_{r^{*}} r_{\theta}^{*}\right]$. 
Proof. See Appendix.

We note that if $\frac{c\left(r^{*}\right)}{(1+r)}$ is constant, then $R_{r^{*}}=0$ and consequently $V_{\theta}^{*}=\bar{a} R_{\theta r}$. Thus the $\operatorname{sign} V_{\theta}^{*}=\operatorname{sign} R_{\theta}$. We use this fact to illustrate the comparative statics in the example below.

Proposition 8. a. Under the conditions of P5 part b, $V_{\lambda}^{*}<0, V_{t}^{*}<0$, and $V_{\delta}^{*}<0$.

b. Under the conditions of P5 part c, $V_{\lambda}^{*}>0, V_{t}^{*}>0$, and $V_{\delta}^{*}>0$.

Proof. See Appendix.

The main conclusion drawn from P8 is that predicting the direction of crime levels based on marginal policy changes is inappropriate. Predictions of crime levels must take into account the various values of all of the parameters in the system. To show that there exist parameter values that satisfy our propositions, we finish with an example.

\section{Example}

This example is meant to capture a situation in which law enforcement is mediocre and the cost of structural improvement is high. Let $t=.4, \lambda=.5, \bar{a}=.5, \gamma=.25$, $\delta=.35, c(r)=c_{1}(1+r)$ and $c_{1}=.556$. It follows that $L=.2$ and $M=.125+.425 r$. Thus for $r<.176$, the conditions for P3 hold, i.e., $0<M<L<\gamma<\bar{a}$. From $\mathrm{P} 4$, we have $\tau_{\bar{a}}^{*}=\frac{1}{2} \frac{.075-.425 r}{.125-.425 r}$ and from P5 we have $\frac{1}{2} \frac{.075-.425 r}{.125-.425 r}=.25$ so $r^{*}=.059$. Substituting, we have $\pi^{*}=.5, \tau_{\bar{a}}^{*}=.25, V^{*}=.125$ and $H\left(V^{*} / \gamma\right)=.5$. Thus, the government described by our example would produce a population in which one-half of the agents would choose to steal more than one-quarter of their firm. Furthermore, because $c\left(r^{*}\right) /\left(1+r^{*}\right)=.556$, it exceeds $\lambda$ and is less than $t+\lambda \delta=.575$. Thus, the conditions of part c of P5 are met. It follows from this that an incremental increase in the probability $\lambda$ of apprehending a thief would result in the undesired outcome of increasing the crime level. 


\section{Appendix}

Proof of P2. If $V \leq 0, \tau_{\bar{a}}^{*}=0$ for all $a$ values and therefore $K(V \mid \gamma)=0$. If $a>V>0$, then $\tau_{\bar{a}}^{*}=V / a$ and the $a$ values satisfying $\tau_{\bar{a}}^{*}>\gamma$ are those satisfying $a<V / \gamma$. The proportion satisfying this inequality, $K(V \mid \gamma)$ will thus equal $H(V / \gamma)$ if $0<V / \gamma<1$ and 1 if $V / \gamma>1$.

Proof of P3. Part a. Since $V=L-\pi M$, it follows that $V \geq L-M$. Thus, by assumption, $V \geq \gamma>0$ and from P2 $K(V \mid \gamma)=1$. Thus, $\pi^{*}$, the probability that G1 will come into existence, must satisfy $\pi=1-K(V \mid \gamma)$, which becomes $\pi^{*}=1-1=0$.

Part b. Since $0<M<L<\gamma$, then again $V>0$. Also, $V=L-\pi M<L$, which by assumption is less than $\gamma$. Thus, $0<V<\gamma$, and from P2, $\pi^{*}$ must satisfy $\pi=1-V / \gamma=1-(L-\pi M) / \gamma$. Solving for $\pi$ yields part b.

Proof of P4. From the proof of P3 part b, we know that $0<V<\gamma$ and since $\gamma<\bar{a}$, we have $0<V<\bar{a}$. Thus, from $\mathrm{P} 1, \tau_{\bar{a}}^{*}=V / \bar{a}=\left(L-\pi^{*} M\right) / \bar{a}=$ $\left[L-\left(\frac{\gamma-L}{\gamma-M}\right) M\right] / \bar{a}=\frac{\gamma}{\bar{a}} \frac{L-M}{\gamma-M}$.

Proof of P5. Part a. From Eq. 2, G1 chooses $r^{*}$ to satisfy $r^{*}=\arg \max _{r_{l} \leq r \leq r_{u}} B_{1}(r)$ such that $(\lambda-t-\lambda \delta) \tau_{\bar{a}}^{*}+t+\lambda \delta \geq c(r) /(1+r)$. By combining terms, $B_{1}(r)$ can be written as $\left.\left.B_{1}(r)=\tau_{\bar{a}}^{*}(1+\lambda r)\right]+\left(1-\tau_{\bar{a}}^{*}\right)(1+r)-\bar{a} \tau_{\bar{a}}^{*^{2}} / 2\right]+k[1-K(V \mid \gamma)]=$ $(1+r)-\tau_{\bar{a}}^{*}(1-\lambda) r-\bar{a} \tau_{\bar{a}}^{*^{2}} / 2+k[1-K(V \mid \gamma)]$. Since $t+\delta \leq 1$ (tax cannot be more than the value of the firm), $M$ is an increasing function of $r$. From P4, it now follows that $\tau_{\bar{a}}^{*}$ is a decreasing function of $r$. Since $\tau_{\bar{a}}^{*}$ is proportional to $V, V$ is a decreasing function of $r$. Therefore, $B_{1}(r)$ is an increasing function of $r$ and it is maximized by the largest value of $r \in\left[r_{l}, r_{u}\right]$ that does not violate the constraint.

Part b. Since $\lambda>t+\lambda \delta$, the left-hand-side of the inequality $(\lambda-t-\lambda \delta) \tau_{\bar{a}}^{*}+t+\lambda \delta$ is a decreasing function of $r$ with value $\lambda$ when $r=r_{l}$ and value $t+\lambda \delta$ when $r=r_{u}$. The right-hand-side, $c(r) /(1+r)$, is non-decreasing and by assumption is less than $\lambda$ 
when $r=r_{l}$ and greater than $t+\lambda \delta$ when $r=r_{u}$. Therefore $r^{*} \in\left(r_{l}, r_{u}\right)$.

Part c. When $\lambda<t+\lambda \delta$, the left-hand-side of the inequality is an increasing function of $r$, with values $\lambda$ and $t+\lambda \delta$ at $r_{l}$ and $r_{u}$ respectively. the right-hand-side is nondecreasing and is larger than $\lambda$ at $r_{l}$ and small than $t+\lambda \delta$ at $r_{u}$. Thus, the largest of the intersections of these curves $r^{*} \in\left(r_{l}, r_{u}\right)$.

Proof of P6. Since $\left.B_{2}(b)=\lambda b+(1-\lambda)\left(1-\tau_{\bar{a}}^{*}\right)() 1-t\right)$ and $\tau_{\bar{a}}^{*}$ does not depend on $b, B_{2}(b)$ is increasing in $b$ and is bounded by 1 .

Proof of P7. Part a. From P3, $\pi_{\theta}^{*}=\frac{-L_{\theta}(\gamma-M)+M_{\theta}(\gamma-L)}{(\gamma-M)^{2}}=\frac{-L_{\theta}}{(\gamma-M)}+\frac{M_{\theta}}{(\gamma-M)} \frac{\gamma-L}{(\gamma-M)}=$ $\frac{1}{(\gamma-M)}\left[L_{\theta}-M_{\theta} \pi^{*}\right]$.

Part b. From P4, $\tau_{\bar{a}_{\theta}}^{*}=\frac{\gamma}{\bar{a}}\left[\frac{\left(L_{\theta}-M_{\theta}\right)(\gamma-M)+M_{\theta}(L-M)}{(\gamma-M)^{2}}\right]=\frac{\gamma}{\bar{a}}\left[\frac{L_{\theta}}{(\gamma-M)}-\frac{M_{\theta}(\gamma-L)}{(\gamma-M)^{2}}=\frac{\gamma}{\bar{a}}\left(\frac{1}{(\gamma-M)}\right)\left[L_{\theta}-\right.\right.$ $\left.M_{\theta} \pi^{*}\right]$.

Part c, Since $\tau_{\bar{a}}^{*}-R\left(\theta, r^{*}\right)=0$, , it follows that $r_{\theta}^{*}=-\frac{\tau_{\bar{\alpha} \theta}^{*}-R_{\theta}}{\tau_{\bar{a} r}^{*}-R_{r^{*}}}$. Substituting, using part $\mathrm{b}$, and clearing fractions, yields part $\mathrm{c}$.

Part d. Since $\tau_{\bar{a}}^{*}=R\left(\theta, r^{*}\right)$, it follows that $\tau_{\bar{a}}^{*}=\bar{a} R\left(\theta, r^{*}\right)$ and the result follows

\section{References}

Acemoglu, Daron, Johnson, Simon and Robinson, James A., 2001. "The Colonial Orgins of Comparative Development: An Empirical Investigation," American Economic Review 91, 1369-1401.

Acemoglu, Daron, Johnson, Simon and Robinson, James A., 2002. "Reversal of Fortune: Geography and Institutions in the Making of the Modern World Income Distribution," Quarterly Journal of Economics 117, 1231-1294.

Acemoglu, Daron, Johnson, Simon and Robinson, James A., 2003. "Disease and Development in Historical Perspective," Journal of the European Economic Association 1, 397-405.

Alexeev, Michael, Janeba, Eckhard and Osborne, Stefan, 2004. "Taxation and 
evasion in the presence of extortion by organized crime," Journal of Comparative Economics 32, 375-387.

Bevan, Alan A. and Estrin, Saul, 2004. "The Determinants of Foreign Direct Investment into European Transition Economies," Journal of Comparative Economics $32,775-787$.

Dixit, Avinash K., 2004. Lawlessness and Economics:Alternative Modes of Governance. Princeton: Princeton University Press.

Djankov, Simeon and Murrell, Peter, 2002. "Enterprise Restructuring in Transition, Journal of Economic Literature, 40, 739-792.

Grossman, Herschel I., 1995. "Rival Kleptocrats: The Mafia Versus the State," in Fiorentini, Gianluca and Peltzman, Sam (Eds.), The Economics of Organized Crime, Cambridge: Cambridge University Press.

Hoff, Karla and Stiglitz, Joseph E., 2004 "After the Big Bang? Obstacles to the Emergence of the Rule of Law in Post-Communist Societies," American Economic Review, 94:3, 753-763.

Katz, Barbara G. and Owen, Joel, 2005. "Moving Toward the Rule of Law in the Face of Corruption: Re-examining the Big-Bang," Stern School of Business Department of Economics Working Paper EC-04-34, revised 8/05.

Katz, Barbara G. and Owen, Joel, 2006. "Crime and Uncertain Punishment in Transition Economies," Stern School of Business Department of Economics Working Paper EC-06-02, revised 12/06.

Kornai, Janos, 2006. "The Great Transformation of Central Eastern Europe: Success and Disappointment," Economics of Transition, 14:2, 207-244

Lotspeich, Richard,1995. "Crime in Transition Economies," Europe-Asia Studies,47:4,555589.

McMillan, John, 2002. Reinventing the Bazaar. New York: W. W. Norton.

Polishchuk, Leonid and Savvateev, Alexei, 2004. "Spontaneous (non)emergence 
of Property Rights," Economics of Transition, 12:1, 103-127.

Shleifer, Andrei and Vishny, Robert W., 1998. The Grabbing Hand: Government Pathologies and Their Cures. Cambridge: Harvard University Press.

Sonin, Konstantin, 2003. "Why the Rich May Favor Poor Protection of Property Rights," Journal of Comparative Economics, 31, 715-731. 